\title{
PROTOCOL AND PRELIMINARY RESULTS OF SYMPTOMS AND CONTROL OF MUCOSITIS IN PATIENTS SUBMITTED TO AUTOLOGOUS STEM CELL TRANSPLANTATION (ASCT).
}

Minicucci, EM'; Cruz, AR ${ }^{2}$; Simões, CC'; Gomes, DO 1; Felipe, DFP1; Gaiola, RD1;

Clinical Hospital of São Paulo State University, Unesp, Medical School, Botucatu, Brazil; 2 Sao Paulo University, Unesp, Medical School Botucatu, Brazil

\section{Introduction:}

Incidence of mucositis in conditioning for hematopoietic stem cell transplant is between 75 and $99 \%$. Objective this study is to present the protocol and preliminary results of symptoms and control of mucositis in patients submitted to autologous stem cell transplantation (ASCT)

\section{Methods:}

From September 2016 to January 2019, the protocol for the prevention of mucositis in patients submitted to ASCT in Clinical Hospital of São Paulo State University, UNESP, Medical School, Botucatu, Brazil, was: children toothpaste, nistatine, daily mouthwash with ice cold chamomile tea, cryotherapy, low level laser therapy, 2J, $660 \mathrm{~nm}, 100 \mathrm{~mW}$, per point in oral cavity and oropharynx, diary starting with mobilization until engraftment of the bone marrow. Sulcralfate was administrated in the first symptoms of dysphagia, clenil $250 \mathrm{cmg}$ spray three times a day at the beginning of mucositis Gll. Mobilization for Multiple Myeloma (MM) was used Melphalan and for lymphoma were used cyclophosphamide, cytarabine, etoposide, carmustine.

Results: Total of patients 34

- $18 \mathrm{MM}$ (10 male, 8 female); 16 lymphoma (8 male, 8 female)

- Incidence of oral mucosits (OM), gastrointesinal mucositis (GIM),

oral and gastrointestinal mucositis $(\mathrm{OM}+\mathrm{GIM})$ in each group.

- Other change observed
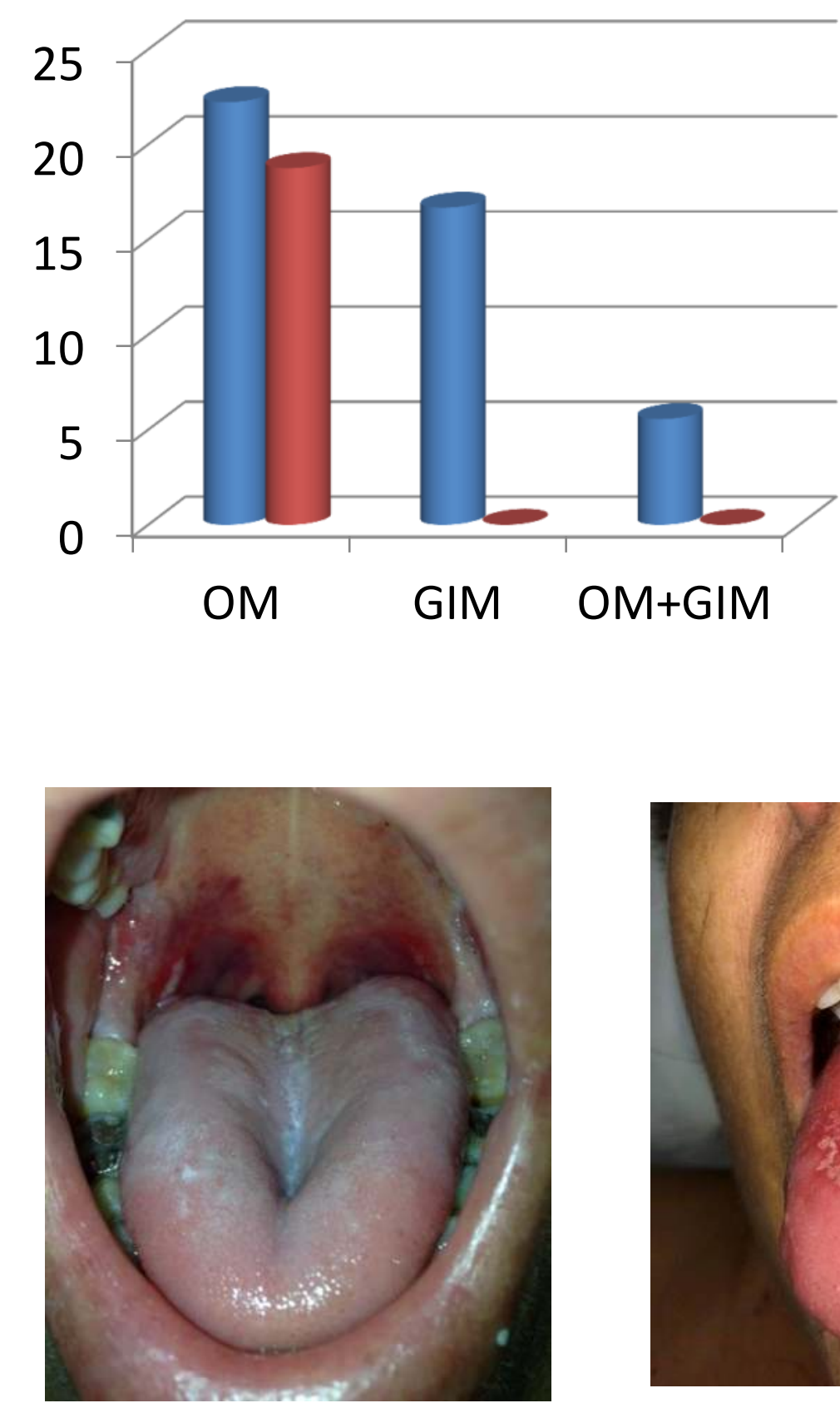

Oral mucositis

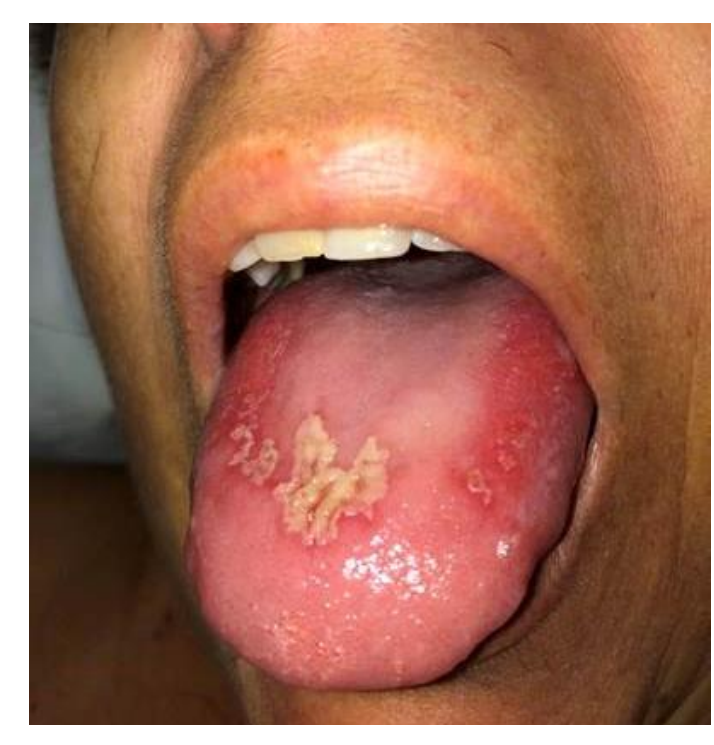

Multiple Mieloma - Lymphoma

.
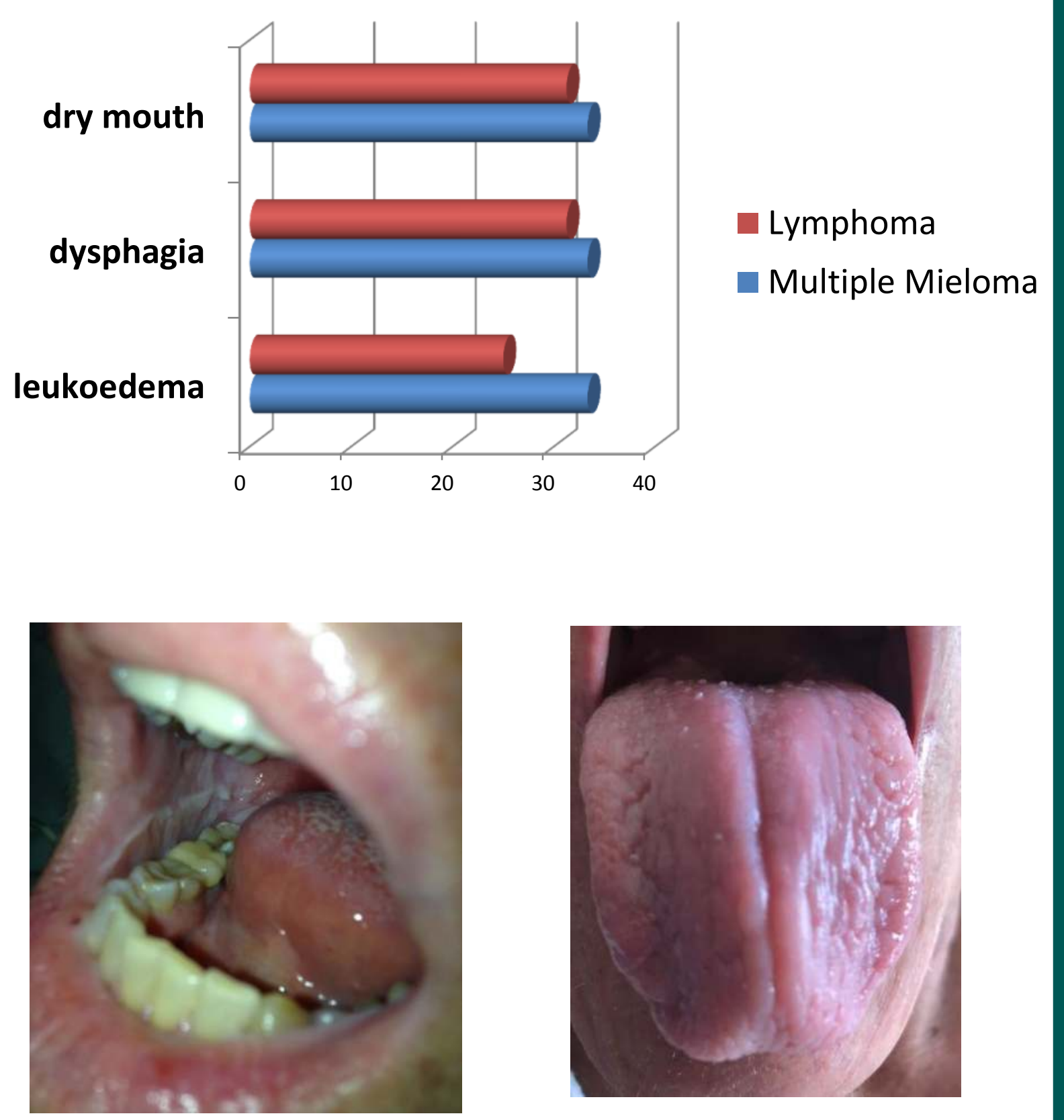

Leukoedema "like"

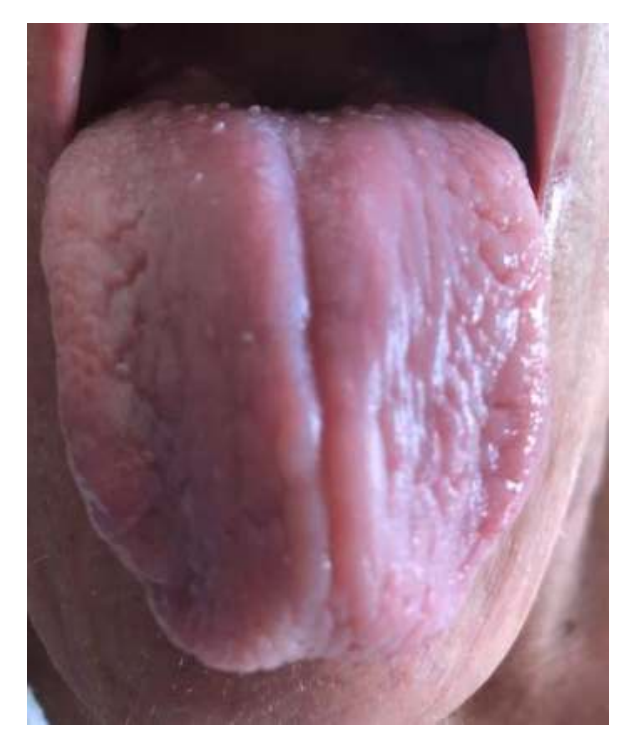

Dry mouth

\section{Conclusion:}

Low incidence of mucositis in ASCT suggest that the protocol seems to be effective in the control of mucositis higher incidence of mucositis, lesions similar leukoedema "like", dry mouth and dysphagia in MM patients comparing with lymphoma, suggesting that the drugs used in MM are more cytotoxic for the mucous. 